\title{
OPEN Meta-analysis of the normal diffusion tensor imaging values of the median nerve and how they change in carpal tunnel syndrome
}

\author{
Djamila Rojoa $\mathbb{\circledR}^{1}$, Firas Raheman ${ }^{(1)}{ }^{1}$, Joseph Rassam ${ }^{1}{ }^{1}$ \& Ryckie G. Wade $\mathbb{1}^{2,3 凶}$
}

Carpal tunnel syndrome (CTS) leads to distortion of axonal architecture, demyelination and fibrosis within the median nerve. Diffusion tensor imaging (DTI) characterises tissue microstructure and generates reproducible proxy measures of nerve 'health' which are sensitive to myelination, axon diameter, fiber density and organisation. This meta-analysis summarises the normal DTI values of the median nerve, and how they change in CTS. This systematic review included studies reporting DTI of the median nerve at the level of the wrist in adults. The primary outcome was to determine the normal fractional anisotropy (FA) and mean diffusivity (MD) of the median nerve. Secondarily, we show how the FA and MD differ between asymptomatic adults and patients with CTS, and how these differences are independent of the acquisition methods. We included 32 studies of 2643 wrists, belonging to 1575 asymptomatic adults and 1068 patients with CTS. The normal FA was $0.58(95 \% \mathrm{Cl} 0.56,0.59)$ and the normal MD was $1.138 \times 10^{-3} \mathrm{~mm}^{2} / \mathrm{s}(95 \% \mathrm{Cl} 1.101,1.174)$. Patients with CTS had a significantly lower FA than controls (mean difference $0.12[95 \% \mathrm{Cl} 0.09,0.16])$. Similarly, the median nerve of patients with CTS had a significantly higher mean diffusivity (mean difference $0.16 \times 10^{-3} \mathrm{~mm}^{2} / \mathrm{s}[95 \% \mathrm{Cl} 0.05$, $0.27]$ ). The differences were independent of experimental factors. We provide summary estimates of the normal FA and MD of the median nerve in asymptomatic adults. Furthermore, we show that diffusion throughout the length of the median nerve becomes more isotropic in patients with CTS.

Carpal tunnel syndrome (CTS) is the most common compressive neuropathy, affecting 10 million people annually. Consequently, CTS is the most expensive upper extremity musculoskeletal disorder, costing the USA health system over $\$ 2$ billion annually and employers up to $\$ 114,000$ per incident ${ }^{1}$.

Compression of peripheral nerves leads to distortion of the axonal architecture, demyelination with or without poor remyelination, loss of the intrinsic vasculature and ultimately, fibrosis of the perineurial and epineurial connective tissue ${ }^{2,3}$. Diffusion tensor imaging (DTI) characterises tissue microstructure and generates reproducible ${ }^{4-8}$ proxy measures of nerve 'health' which are sensitive to myelination, axon diameter, fibre density and organisation ${ }^{9-11}$ (Fig. 1). DTI typically generates the following metrics: fractional anisotropy (FA), mean diffusivity (MD), axial diffusivity ( $\mathrm{AD}$ ) and radial diffusivity $(\mathrm{RD})$. FA is a scalar value between zero and one-a FA of zero implies isotropic diffusion of water within a voxel, whilst a FA of one implies diffusion along a single axis (i.e., bidirectional diffusion along the length of the nerve). MD describes the average molecular diffusion rate within the voxel, whilst $\mathrm{AD}$ describes diffusion in the long axis and $\mathrm{RD}$ represents diffusion perpendicular to the long axis.

Several studies have shown that DTI metrics (FA and MD) are sensitive to microstructural changes which occur within the median nerve of patients with CTS (Fig. 1). However, there are several uncertainties that must be resolved before this technology could be used in clinical practice or as a reference standard in research studies. Firstly, the normal DTI values of the median nerve must be established and secondly, uncertainty around how experimental conditions (e.g. scanning parameters) influence DTI metrics need to be determined. These uncertainties, and how DTI metrics change in CTS, might be resolved through meta-analysis and represents the rationale for this study.

\footnotetext{
${ }^{1}$ Department of Plastic and Reconstructive Surgery, Leicester Royal Infirmary, Leicester, UK. ${ }^{2}$ Department of Plastic and Reconstructive Surgery, Leeds Teaching Hospitals Trust, Leeds, UK. ${ }^{3}$ Leeds Institute for Medical Research, Advanced Imaging Centre, University of Leeds, Leeds LS1 3EX, UK. ${ }^{凶}$ email: ryckiewade@gmail.com
} 

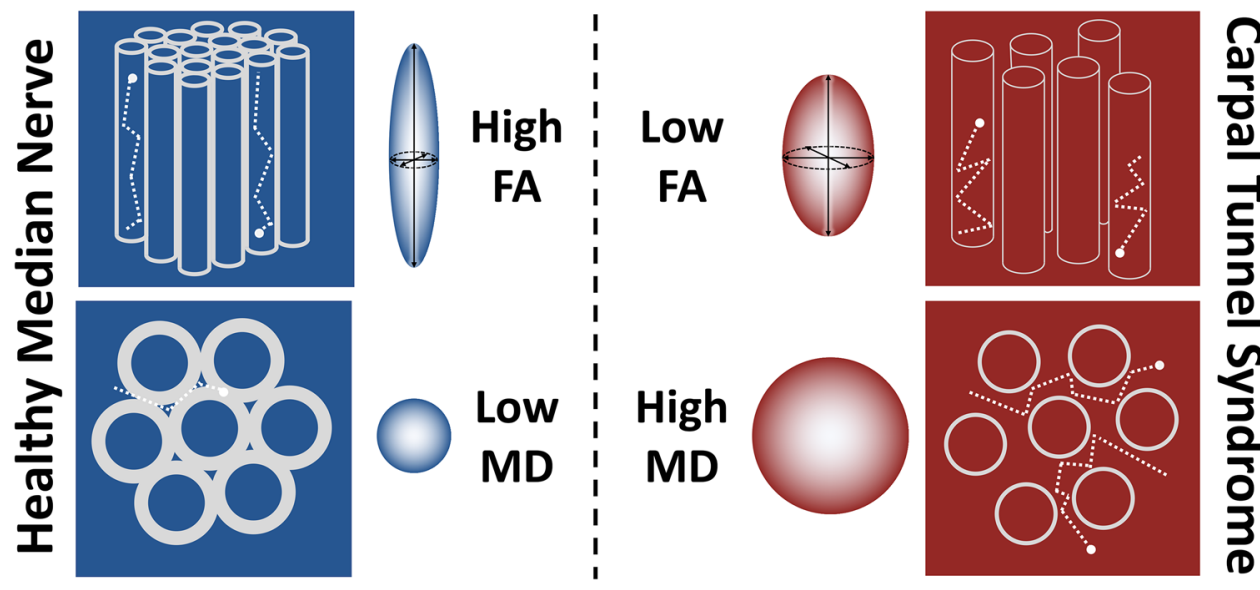

Figure 1. A diagram of nerve fibres (top) and in cross-section (bottom) demonstrating how diffusion tensor imaging metrics change in CTS. In healthy nerves, the axons are enveloped by myelin sheaths and arranged relatively tightly, which restricts the diffusion of water to the long axis of the nerve. Chronic compression leads to distortion of the axonal architecture, demyelination and as such, degradation of physiological barriers to the diffusion of water diffusion. Consequently, more diffusion occurs perpendicular to the long axis of the nerve as water is more free to diffuse around the fibres, reducing the factional anisotropy (FA) and increasing the magnitude of diffusion (mean diffusivity, MD).

\section{Methods}

This review is registered with PROSPERO (CRD42020212378). It was designed and conducted in accordance with the Cochrane Handbook of Systematic Reviews ${ }^{12}$ and has been authored in accordance with the PRISMA 2020 statement $^{13}$.

Types of studies. We included all studies which reported the findings of diffusion tensor magnetic resonance imaging of the median nerve, at the level of the wrist in asymptomatic adults or adults with CTS. There were no language restrictions. We excluded case reports and studies which did not report DTI metrics (e.g., studies which contained fibre tractography graphics only) of the median nerve.

Participants. This review considers 2 distinct populations:

1. Asymptomatic adults (aged $\geq 16$ years) with no known pathology (past or present) affecting the peripheral nerves of the upper limb.

2. Adults with a diagnosis of carpal tunnel syndrome. For a study to be included, we did not impose any specific thresholds or criteria on the diagnosis of CTS, such as the presence of specific symptoms, provocative tests, aberrant electrophysiological parameters or imaging features.

Search strategy. The NICE Healthcare Databases (hdas.nice.org.uk) was searched according to Appendix 1 (Supplementary Materials) on 9th October 2020. The medRxiv and bioRxiv preprint archives were searched with the same strategy using the R package medrxivr ${ }^{14}$. Later, CitationChaser ${ }^{15}$ was used for forward and backward citation chasing based on the final list of included studies (eFigure 1).

Study selection. Three review authors (DR, JR and FR) independently screened titles and abstracts for relevance, in accordance with the eligibility criteria. The full texts of potentially eligible articles were obtained and again independently assessed by the same authors. Disagreements were resolved by discussion with RGW. The reasons for excluding studies are outlined in Appendix 2 (Supplementary Materials).

Data extraction. Three review authors (DR, JR and FR) independently double extracted all data. Thereafter, all datapoints were independently checked for accuracy by RGW. DTI parameters were extracted from the following anatomical levels of the median nerve: the distal radio-ulnar joint (DRUJ), the pisiform and the hook of the hamate, as these are three commonly used imaging landmarks which equate to the inlet, mid-point and outlet of the carpal tunnel. The nerve/hand was the unit of analysis ${ }^{16}$. Many studies reported both the number of individuals and wrists scanned (as some studies involved bilateral scanning) but if not otherwise stated we assumed imaging was performed unilaterally. If data were missing, unclear or present in an unfavourable format then the authors were contacted by email with a request for more information. Four authors provided additional information upon request ${ }^{17-20}$. When no reply was received, estimates were derived from graphs or imputed where possible ${ }^{21}$. 
Outcomes. The primary outcome was to estimate the normal DTI metrics (FA and MD) of the median nerve in asymptomatic adults. The secondary outcomes were to estimate the differences in DTI metrics (FA and MD) between asymptomatic adults and patients with CTS, and explore the associations between DTI metrics and: age, echo time (TE), repetition time (TR), resolution, the number of diffusion sensitising gradient directions $\left(\mathrm{N}_{\mathrm{D}}\right)$ sampled per shell, the b-value(s), different methods of $\mathrm{k}$-space sampling and in-plane acceleration.

Methodological quality assessment. The risk of bias was independently assessed by three review authors (DR, JR and FR) using the ROBINS-I tool ${ }^{22}$ and displayed graphically using robvis ${ }^{23}$. Disagreements were resolved by discussion with RGW.

Statistical analysis. The raw data are available via the Open Science Framework (https://osf.io/vqwkp/). The single study performed at 7 tesla ${ }^{19}$ was excluded from all meta-analyses given its clinical disparity. Using the meta suite of Stata v16 (StataCorp, Texas), the mean FA and MD from asymptomatic adults were pooled to estimate the normative values. We performed direct comparisons meta-analysis of the mean differences in FA and MD between asymptomatic adults and patients with CTS. Meta-analyses were subgrouped by anatomical location. Restricted maximum likelihood was used to estimate the between-study variance $\left(\operatorname{tau}^{2}\right)$, with the Knapp and Hartung modification. Heterogeneity was quantified by $\mathrm{I}^{2}{ }^{24}$.Using the metafor ${ }^{25}$ package, mixed-effects meta-regression was used to explore potential reasons for the observed heterogeneity in the direct comparisons meta-analysis of FA; the continuous covariates were age, in-plane resolution $\left(\mathrm{mm}^{2}\right)$, slice thickness ( $\left.\mathrm{mm}\right)$, echo time (TE in $\mathrm{ms}$ ), b-value $\left(\mathrm{mm}^{2} / \mathrm{s}\right)$ and number of diffusion-sensitising gradient directions $\left(\mathrm{N}_{\mathrm{D}}\right)$. TE and b-value were modelled as an interaction. Confidence intervals (CI) were generated to the $95 \%$ level. To investigate the possibility of small-study effects for FA between asymptomatic adults and patients with CTS, a funnel plot was constructed with the pseudo CIs contoured by $\operatorname{tau}^{22}$. EviAtlas was used to generate a map of the location of the 1 st author's institution ${ }^{26}$.

\section{Results}

Ultimately, 32 studies ${ }^{9,17-20,27-53}$ were included (eFigure 2).

Study characteristics. Study characteristics are detailed in eTable 1. Overall, we included data from 2643 wrists belonging to 1575 asymptomatic adults and 1068 patients with CTS. Asymptomatic adults were a mean 6 years younger than patients with CTS $(95 \%$ CI 3,10$)$. There were approximately twice as many females (1404:746) although this disparity was more pronounced in patients with CTS (660F:193 M) than asymptomatic adults (737F:552 M). The median number of authors was 6 (IQR 5-8) and studies were derived from 16 different countries (eFigure 3).

Ten studies (32\%) were performed at a field strength of 1.5 tesla $18,30,33,36,39,43,45,49,50,52$, twenty one (65\%) at 3 tesla $\mathrm{a}^{9,17,20,27-29,31,32,34,35,37,38,40-42,44,46-48,51,53}$, and one at 7 tesla $^{19}$. The median echo and repetition times were $87 \mathrm{~ms}$ (IQR 65-91, range 21-103) and $7000 \mathrm{~ms}$ (IQR 3800-7650, range 1470-10,254), respectively. Two studies used read-out segment echo-planar imaging (rsEPI) ${ }^{34,46}$, two did not specify ${ }^{20,48}$ and the remainder used singleshot echo-planar imaging (ssEPI). Twelve studies described in-plane acceleration techniques (GRAPPA ${ }^{19,28,42}$, SENSE $^{18,29,32,39,41,46,51}$ and CAIPIRINHA $\left.{ }^{31}\right)$ and six studies used partial Fourier transformations ${ }^{31,33,35,39,41,44,46}$. The median slice thickness was $3.5 \mathrm{~mm}$ (IQR 2.6-4.0 $\mathrm{mm}$, range $1.5-5 \mathrm{~mm}$ ). The median in-plane resolution was $1.09 \mathrm{~mm}$ (IQR $0 \cdot 7-1 \cdot 5$, range $0 \cdot 4-1 \cdot 88$ ). Two studies investigated multiple b-values ${ }^{36,51}$ via discrete shells, although no studies reported whether acquisitions were half or whole shell and what sample scheme was used. The mean b-value was $1000 \mathrm{~s} / \mathrm{mm}^{2}$ (SD 270, range 325-2000). The median $\mathrm{N}_{\mathrm{D}}$ was 20 (IQR 15-25, range 6-32). A median of 3 signal averages (excitations) were obtained (IQR 2-5, range 1-12). When reported, the mean SNR of the b0 images was 25 (SD 12) $)^{19,29,31,33,36,39}$.

The risk of bias for the included studies is summarised in eFigure 4 . The majority of studies were at low risk of methodological bias.

Evidence synthesis: asymptomatic adults. The FA of the median nerve in asymptomatic adults was reported in 29 studies $9,17-20,27-36,38-42,44-51,53$. Overall, the normal FA was 0.58 (95\% CI 0.56, 0.59; I ${ }^{2} 98 \%$ ). The FA was highest at the level of the DRUJ (mean 0.61 [95\% CI 0.58, 0.63]; $\mathrm{I}^{2} 96 \%$ ), dropping at the level of the pisiform to 0.57 (95\% CI $\left.0.54,0.61 ; \mathrm{I}^{2} 98 \%\right)$ and lowest at the level of the hook of the hamate (mean 0.54 [95\% CI 0.51, 0.57]; I $\left.{ }^{2} 95 \%\right)$.

The MD of the median nerve in asymptomatic adults was reported in 28 studies ${ }^{9,17-20,27-36,38-42,44-51}$. Overall, the normal MD was $1.138 \times 10^{-3} \mathrm{~mm}^{2} / \mathrm{s}$ (95\% CI 1.101, 1.174; $\left.\mathrm{I}^{2} 99 \%\right)$. The MD was lowest at the level of the DRUJ (mean $1.073 \times 10^{-3} \mathrm{~mm}^{2} / \mathrm{s}$ [95\% CI 1.019, 1.128]; $\mathrm{I}^{2} 93 \%$ ), increasing at the level of the pisiform (mean $1.180 \times 10^{-3} \mathrm{~mm}^{2} / \mathrm{s}[95 \%$ CI $\left.1.115,1.244] ; \mathrm{I}^{2} 96 \%\right)$ and highest at the level of the hook of the hamate (mean $1.151 \times 10^{-3} \mathrm{~mm}^{2} / \mathrm{s}[95 \%$ CI $\left.1.086,1.217] ; \mathrm{I}^{2} 98 \%\right)$.

Evidence synthesis: patients with CTS. The FA of the median nerve in patients with CTS was reported in 19 studies $^{17-20,27-29,32-34,38-40,45,47-50,52}$. Overall, patients had a mean FA of 0.45 (95\% CI 0.43, 0.47; $\left.\mathrm{I}^{2} 95 \%\right)$. The FA was lowest at the mid-point of carpal tunnel, at the level of the pisiform (mean 0.41 [95\% CI 0.38, 0.43]; $\mathrm{I}^{2}$ 86\%), compared to the levels of the DRUJ (mean 0.48 [95\% CI 0.44, 0.52]; $\mathrm{I}^{2} 91 \%$ ) or hook of the hamate (mean 0.45 [95\% CI 0.42-0.48]; I I 93\%).

The MD of the median nerve in patients with CTS was reported in 18 studies $^{17-20,27-29,32-34,39,40,45,47-50,52}$. Overall, patients with CTS had a pooled mean MD of $1.293 \times 10^{-3} \mathrm{~mm}^{2} / \mathrm{s}$ (95\% CI 1.227, 1.359; $\left.\mathrm{I}^{2} 99 \%\right)$. The MD 
was highest at the level of the pisiform (mean $1.372 \times 10^{-3} \mathrm{~mm}^{2} / \mathrm{s}$ [95\% CI $\left.1.245-1.500\right] ; \mathrm{I}^{2} 98 \%$ ), $1.180 \times 10^{-3}$ $\mathrm{mm}^{2} / \mathrm{s}$ at the level of the DRUJ $\left(95 \%\right.$ CI $\left.1.064,1.295 ; \mathrm{I}^{2} 95 \%\right)$ and $1.335 \times 10^{-3} \mathrm{~mm}^{2} / \mathrm{s}$ at the level of the hook of the hamate (95\% CI 1.259, 1.411; I $\left.\mathrm{I}^{2} 93 \%\right)$.

Direct comparisons meta-analysis: asymptomatic adults vs. patients with CTS. Fourteen studies reported direct comparisons between asymptomatic adults and patients with CTS ${ }^{17-19,28,29,32-34,39,40,45,47,49,50}$. All studies reported a lower FA in patients with CTS compared to asymptomatic adults (mean difference 0.09 [95\% CI 0.07, 0.11]; Fig. 2). The largest difference between asymptomatic adults and patients with CTS was at the mid-point of the carpal tunnel, at the level of the pisiform (mean difference 0.12 [95\% CI 0.09, 0.16]).

Patients with CTS had a higher mean diffusivity than asymptomatic adults (mean difference $0.12 \times 10^{-3} \mathrm{~mm}^{2} / \mathrm{s}$ [95\% CI 0.08, 0.17], Fig. 3). This difference was again most profound at the mid-point of the carpal tunnel, at the level of the pisiform (mean difference $0.16 \times 10^{-3} \mathrm{~mm}^{2} / \mathrm{s}$ [95\% CI 0.05, 0.27]).

Meta-regression. Age was negatively associated with the FA in asymptomatic adults whereby each decade of life reduced the FA by approximately 0.003 (adjusted $\beta-2.79 \times 10^{-3}\left[95 \% \mathrm{CI}-4.78 \times 10^{-3},-8.12 \times 10^{-4}\right] ; \mathrm{I}^{2}$ 97\%). However, there was no relationship between age and FA in patients with CTS (adjusted $\beta 9.70 \times 10^{-4}[95 \%$ $\mathrm{CI}-2.89 \times 10^{-3}, 4.83 \times 10^{-3} ; \mathrm{I}^{2} 96 \%$ ], Fig. 4). Increasing age was also associated with MD whereby each decade of life increased MD by approximately $0.108 \times 10^{-4} \mathrm{~mm}^{2} / \mathrm{s}\left(95 \%\right.$ CI $0.073 \times 10^{-4}, 0.140 \times 10^{-4} ; \mathrm{I}^{2} 99 \%$, Fig. 5) with no significant difference between asymptomatic adults and patients with CTS.

There was no relationship between $\mathrm{N}_{\mathrm{D}}$ and FA (eFigure 5) or MD (eFigure 66). The b-value was not associated with the FA (eFigure 7). There was an inverse relationship between the b-value and MD, whereby increments of $100 \mathrm{~mm}^{2} / \mathrm{s}$ reduced the mean diffusivity by $0.04 \times 10^{-3} \mathrm{~mm}^{2} / \mathrm{s}\left(\beta-3.849 \times 10^{-7} \mathrm{~mm}^{2} / \mathrm{s}\left[95 \% \mathrm{CI}-5.019 \times 10^{-7}\right.\right.$, $\left.-2.678 \times 10^{-7}\right] ; \mathrm{I}^{2} 98 \%$, eFigure 8 ).

There were no significant differences between studies which used ssEPI or rsEPI. There was no association between the in-plane resolution (in square millimetres) and FA (eFigure 9) or MD (eFigure 10). Slice thickness was not associated with FA (eFigure 11) but was negatively associated with MD whereby increments of $1 \mathrm{~mm}$ reduced the MD by $6.023 \times 10^{-5} \mathrm{~mm}^{2} / \mathrm{s}\left(95 \%\right.$ CI $9.754 \times 10^{-5}, 2.294 \times 10^{-5} ; \mathrm{I}^{2} 99 \%$, eFigure 12$)$.

The TE was not associated with FA or MD (eFigures 13 and 14). The TR was not associated with FA (eFigure 15) but longer repetition times were associated with lower estimates of $\mathrm{MD}$, whereby increasing the TR by $1 \mathrm{~s}$ decreased the MD by $2.990 \times 10^{-6} \mathrm{~mm}^{2} / \mathrm{s}\left(95 \%\right.$ CI $4.383 \times 10^{-6}, 1.598 \times 10^{-6}$; eFigure 16$)$.

Studies reporting the use of parallel imaging techniques (e.g. GRAPPA, SENSE or ASSET) yielded 5\% higher estimates of FA ( $\beta 0.05$ [95\% CI 0.02, 0.08]; $\mathrm{I}^{2}$ 98\%, eFigure 17) when compared to studies which did not report this information. Parallel imaging methods were not associated with differences in the MD. There was insufficient data to explore different partial Fourier settings. There was no association between the number of signal averages and FA or MD (eFigures 18 and 19).

Ultimately, mixed-effects multivariable meta-regression showed that having CTS was the strongest independent moderator of the observed heterogeneity in FA (Table 1). Age explained some of the residual between-study variance. The experimental factors we modelled did not explain the residual heterogeneity.

There was no evidence of publication bias (Eggers $\beta 0.10$ [95\% CI 0.06, 0.14]; $\mathrm{p}=0.134$, eFigure 20).

\section{Discussion}

This study demonstrates that throughout the length of the median nerve at the wrist, patients with CTS have more isotropic diffusion than asymptomatic adults. The largest differences for both fractional anisotropy and mean diffusivity were observed at the mid-point of the carpal tunnel, at the level of pisiform where CTS patients had lower FA and higher MD. Of clinical importance, we demonstrate that these real-world differences were independent of age and experimental (acquisition) conditions. Therefore, we believe that aberrations in both FA and diffusivity could be used to identify patients with median nerve neuropathy at the wrist.

There are inherent problems with clinicians diagnosing CTS given that the constellation of symptoms and clinical signs of the syndrome, and the available tests are largely unreliable. For example, nocturnal paraesthesias and many classical tests such as Phalen and Tinel, the scratch-collapse and sensory threshold testing have poor diagnostic value ${ }^{54,55}$. Despite the widespread use of electrodiagnostic studies in patients with suspected carpal tunnel syndrome, it remains an invasive test which evokes pain and anxiety, and controversy still exists regarding its accuracy and the normal values ${ }^{56,57}$. For these reasons, surgeons still perform decompression surgery in the presence of normal provocative and electrodiagnostic tests ${ }^{58}$. More recently, measurement of the cross sectional area of the median nerve using ultrasound is gaining popularity given that it has good inter-rater and intra-rater reliability ${ }^{59}$, and validity ${ }^{60,61}$. Despite these benefits, ultrasound has not been adopted into routine clinical practice because several aspects remain unclear, such as (i) how the cross-sectional area is affected by other factors such as age, sex, diabetes, sonographer technique [pressure applied, measurement angle, etc.] and the hardware, (ii) whether the epineurium should be included in the measurement, and (iii) how these measurements relate to severity, subjective and objective outcomes. Moreover, sonographically derived cross-sectional area still only provides morphological information (size and shape) which is inherently insensitive to nerve function and microstructure. Therefore, considerable effort has been directed towards the development of DTI because it characterises tissue microstructure and generates reproducible ${ }^{4-8}$ proxy measures of nerve 'health' which are sensitive to myelination, axon diameter, fibre density and organisation ${ }^{9-11}$ (Fig. 1). DTI metrics outperform standard morphological imaging in patients with peripheral neuropathy ${ }^{35}$ and are independent of age and acquisition parameters, something which cannot be said for electrodiagnostics ${ }^{62}$ or sonography. In the UK, the cost of a non-contrast MRI of the extremity is less than an electrodiagnostic exam ( $£ 389$ versus $£ 444$ ) but more than sonography $(£ 220)^{63}$. And DTI would be supplemented with other MRI data, such as morphological 
Fractional Anisotropy Reduces in Carpal Tunnel Syndrome

\begin{tabular}{|c|c|c|c|c|c|c|}
\hline Study & b-Value $\left(\mathrm{s} / \mathrm{mm}^{2}\right)$ & Directions & Tesla & & $\begin{array}{c}\text { Weight } \\
(\%)\end{array}$ & $\begin{array}{c}\text { Mean Diff. } \\
\text { with } 95 \% \mathrm{Cl}\end{array}$ \\
\hline \multicolumn{7}{|l|}{ Distal Radioulnar Joint } \\
\hline Wang 2012 & 400 & 32 & 1.5 & & 3.37 & $-0.06[-0.09,-0.02]$ \\
\hline Cingoz 2018 & 800 & 20 & 1.5 & & 2.33 & $-0.04[-0.12,0.04]$ \\
\hline Stein 2009 & 1000 & 6 & 3 & & 3.08 & $-0.04[-0.09,0.01]$ \\
\hline Koh 2014 & 1000 & 15 & 3 & & 3.19 & $-0.08[-0.12,-0.04]$ \\
\hline Barcelo 2013 & 1000 & 15 & 3 & & 0.85 & $-0.03[-0.23,0.16]$ \\
\hline Ta?delen 2011 & 1000 & 16 & 3 & & 3.44 & $-0.12[-0.15,-0.09]$ \\
\hline Hiltunen 2012 & 1000 & 25 & 3 & & 0.78 & $-0.04[-0.24,0.17]$ \\
\hline Paniandi 2018 & 1000 & 32 & 3 & & 1.83 & $-0.06[-0.17,0.04]$ \\
\hline Wafaie 2018 & 1000 & 32 & 1.5 & & 3.38 & $-0.13[-0.16,-0.10]$ \\
\hline Kabakci 2007 & 1000 & 32 & 3 & & 2.66 & $-0.28[-0.35,-0.22]$ \\
\hline Guggenberger 2012 & 1200 & 15 & 3 & & 2.92 & $-0.06[-0.12,-0.00]$ \\
\hline \multicolumn{5}{|c|}{ Heterogeneity: $\tau^{2}=0.00, \mathrm{I}^{2}=86.52 \%, \mathrm{H}^{2}=7.42$} & & $-0.09[-0.14,-0.05]$ \\
\hline \multicolumn{7}{|c|}{ Test of $\theta_{i}=\theta_{j}: Q(10)=53.17, p=0.00$} \\
\hline \multicolumn{7}{|l|}{ Pisiform } \\
\hline Wang 2012 & 400 & 32 & 1.5 & & 3.37 & $-0.06[-0.09,-0.02]$ \\
\hline Cingoz 2018 & 800 & 20 & 1.5 & & 3.05 & $-0.17[-0.22,-0.12]$ \\
\hline Stein 2009 & 1000 & 6 & 3 & & 3.08 & $-0.12[-0.17,-0.07]$ \\
\hline Brienza 2014 & 1000 & 6 & 3 & & 3.42 & $-0.23[-0.26,-0.20]$ \\
\hline Koh 2014 & 1000 & 15 & 3 & & 3.43 & $-0.10[-0.13,-0.06]$ \\
\hline Kwon 2015 & 1000 & 15 & 3 & & 3.29 & $-0.11[-0.15,-0.07]$ \\
\hline Barcelo 2013 & 1000 & 15 & 3 & & 1.19 & $-0.17[-0.32,-0.01]$ \\
\hline Hiltunen 2012 & 1000 & 25 & 3 & & 2.06 & $-0.05[-0.15,0.04]$ \\
\hline Paniandi 2018 & 1000 & 32 & 3 & & 2.50 & $-0.04[-0.12,0.03]$ \\
\hline Wafaie 2018 & 1000 & 32 & 1.5 & & 3.36 & $-0.18[-0.21,-0.15]$ \\
\hline Guggenberger 2012 & 1200 & 15 & 3 & & 3.12 & $-0.10[-0.15,-0.05]$ \\
\hline \multicolumn{4}{|c|}{ Heterogeneity: $\tau^{2}=0.00, \mathrm{I}^{2}=86.13 \%, \mathrm{H}^{2}=7.21$} & & & $-0.12[-0.16,-0.09]$ \\
\hline \multicolumn{7}{|c|}{ Test of $\theta_{i}=\theta_{j}: Q(10)=84.62, p=0.00$} \\
\hline \multicolumn{7}{|l|}{ Hook of the Hamate } \\
\hline Wang 2012 & 400 & 32 & 1.5 & & 3.46 & $-0.04[-0.07,-0.02]$ \\
\hline Cingoz 2018 & 800 & 20 & 1.5 & & 2.49 & $-0.02[-0.10,0.05]$ \\
\hline Stein 2009 & 1000 & 6 & 3 & & 3.21 & $-0.08[-0.12,-0.04]$ \\
\hline Klauser 2017 & 1000 & 12 & 3 & & 3.11 & $-0.08[-0.12,-0.03]$ \\
\hline Koh 2014 & 1000 & 15 & 3 & & 3.45 & $-0.04[-0.07,-0.01]$ \\
\hline Kwon 2015 & 1000 & 15 & 3 & & 3.42 & $-0.04[-0.07,-0.01]$ \\
\hline Razek 2016 & 1000 & 15 & 1.5 & & 3.41 & $-0.05[-0.08,-0.02]$ \\
\hline Barcelo 2013 & 1000 & 15 & 3 & $\rightarrow$ & 1.09 & $-0.21[-0.37,-0.04]$ \\
\hline Hiltunen 2012 & 1000 & 25 & 3 & & 1.64 & $0.00[-0.12, \quad 0.12]$ \\
\hline Paniandi 2018 & 1000 & 32 & 3 & & 1.84 & $-0.05[-0.15,0.06]$ \\
\hline Wafaie 2018 & 1000 & 32 & 1.5 & & 3.29 & $-0.16[-0.20,-0.12]$ \\
\hline Guggenberger 2012 & 1200 & 15 & 3 & & 3.08 & $-0.06[-0.11,-0.01]$ \\
\hline \multicolumn{5}{|c|}{ Heterogeneity: $\tau^{2}=0.00, I^{2}=71.03 \%, H^{2}=3.45$} & & $-0.06[-0.09,-0.04]$ \\
\hline \multicolumn{7}{|c|}{ Test of $\theta_{i}=\theta_{j}: Q(11)=37.40, p=0.00$} \\
\hline \multicolumn{7}{|l|}{ Unspecified } \\
\hline Khalil 2008 & 400 & 32 & 1.5 & & 3.14 & $-0.07[-0.12,-0.03]$ \\
\hline Bulut 2014 & 1000 & 32 & 1.5 & & 3.67 & $-0.06[-0.07,-0.05]$ \\
\hline \multicolumn{4}{|c|}{ Heterogeneity: $\tau^{2}=0.00, I^{2}=0.08 \%, H^{2}=1.00$} & & & $-0.06[-0.07,-0.05]$ \\
\hline \multicolumn{7}{|c|}{ Test of $\theta_{i}=\theta_{j}: Q(1)=0.38, p=0.54$} \\
\hline \multicolumn{5}{|l|}{ Overall } & & $-0.09[-0.11,-0.07]$ \\
\hline \multicolumn{7}{|c|}{ Heterogeneity: $\tau^{2}=0.00, I^{2}=87.72 \%, H^{2}=8.14$} \\
\hline \multicolumn{7}{|c|}{ Test of $\theta_{i}=\theta_{j}: Q(35)=258.52, p=0.00$} \\
\hline \multicolumn{7}{|c|}{ Test of group differences: $Q_{b}(3)=10.78, p=0.01$} \\
\hline & & & -.4 & -.2 & .2 & \\
\hline
\end{tabular}

Figure 2. A forest plot of the fractional anisotropy of the median nerve, at 3 anatomical levels, between asymptomatic adults and patients with carpal tunnel syndrome. 
Mean Diffusivity Increases in Carpal Tunnel Syndrome

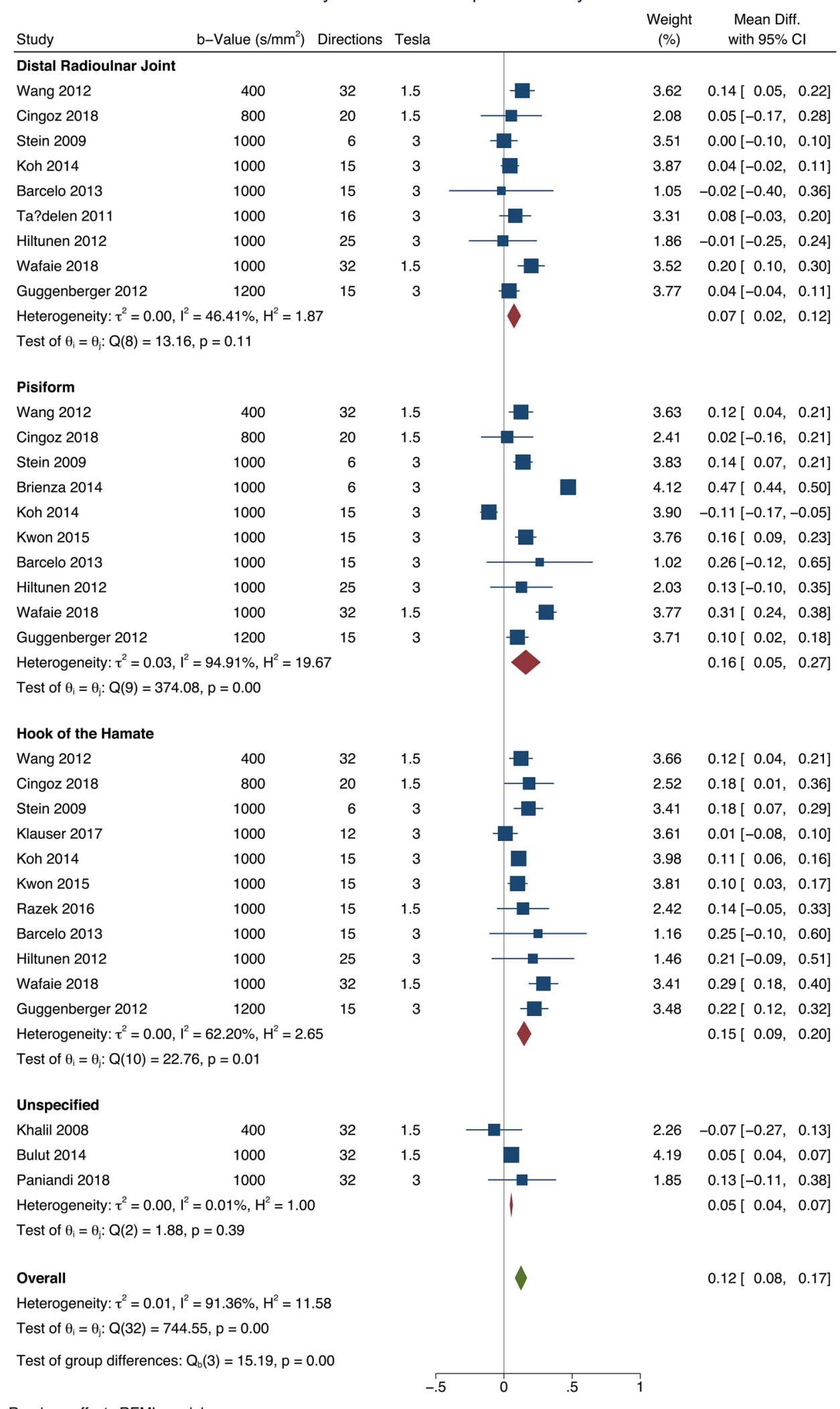

Random-effects REML model

Sorted by: bval directions

Figure 3. A forest plot of the mean diffusivity of the median nerve, at 3 anatomical levels, between asymptomatic adults and patients with carpal tunnel syndrome. 


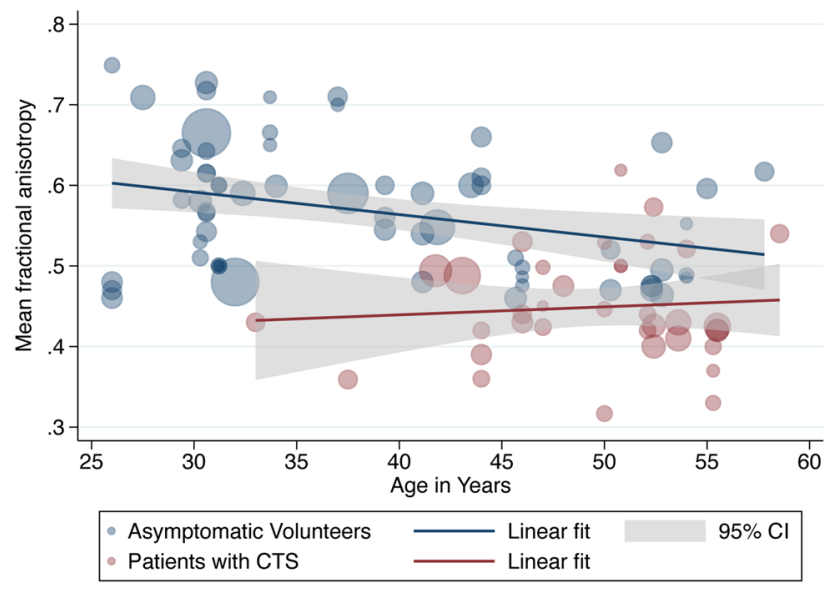

Figure 4. A scatterplot of study-level estimates of fractional anisotropy in asymptomatic adults and patients with carpal tunnel syndrome, against age in years. The size of the points corresponds to the precision (inverse variance) of the study.

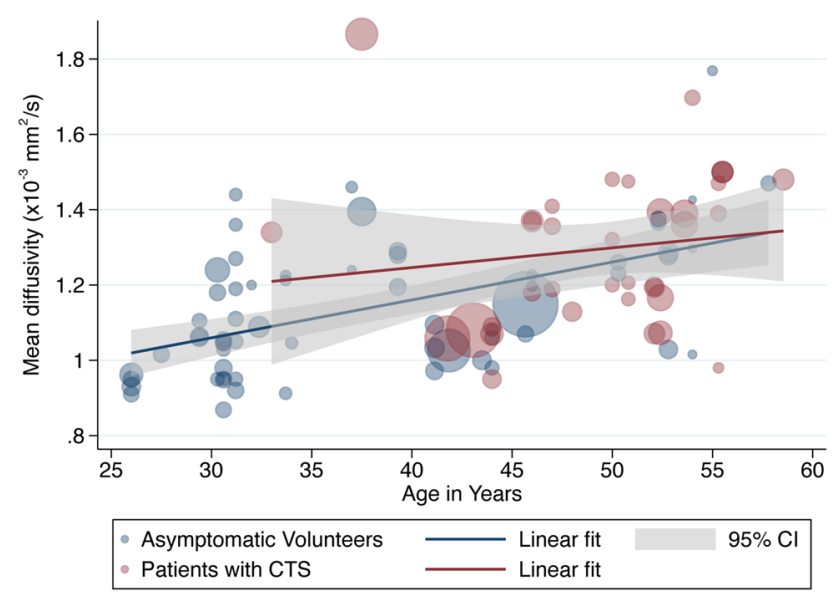

Figure 5. A scatterplot of study-level estimates of mean diffusivity in asymptomatic adults and patients with carpal tunnel syndrome, against age in years. The size of the points corresponds to the precision (inverse variance) of the study.

\begin{tabular}{|l|l|l|l|}
\hline Covariates & $\begin{array}{l}\text { Adjusted change in fractional anisotropy } \\
(\boldsymbol{\beta})\end{array}$ & $\mathbf{9 5 \%} \mathbf{C I}$ & Resampled p-value \\
\hline Patients with carpal tunnel syndrome & $-8.57 \times 10^{-2}$ & $-0.13,-0.06$ & 0.000 \\
\hline Age in years & $-2.92 \times 10^{-3}$ & $-4.54 \times 10^{-3},-7.34 \times 10^{-4}$ & 0.007 \\
\hline Repetition time (ms) & $-8.98 \times 10^{-6}$ & $-1.14 \times 10^{-5}, 6.77 \times 10^{-8}$ & 0.053 \\
\hline $\begin{array}{l}\text { Number of diffusion sensitising gradient } \\
\text { directions }\end{array}$ & $2.27 \times 10^{-3}$ & $-8.39 \times 10^{-4}, 2.99 \times 10^{-3}$ & 0.271 \\
\hline Echo time $(\mathrm{ms})$ and b-value $\left(\mathrm{mm}^{2} / \mathrm{s}\right)^{*}$ & $-1.51 \times 10^{-8}$ & $-5.27 \times 10^{-7}, 4.86 \times 10^{-7}$ & 0.936 \\
\hline
\end{tabular}

Table 1. Mixed-effects meta-regression. Adjusted $\mathrm{R}^{2}=46 \%, \operatorname{tau}^{2}=0 \cdot 0047, \mathrm{I}^{2}=97 \%$. ${ }^{\star}$ Echo time is a function of the $b$-value as larger b-values mandate relatively longer echo times, so these variables are modelled as the product to minimise the number of covariables, mitigate collinearity and model the interaction between the two variables. 
(anatomical) scans, contrast-free angiography, sequences which characterise muscle (fat fractions, elastography, iron deposition, etc.) and the topography of the sensorimotor cortex (e.g., using functional MRI) to determine whether there is central capacity to regenerate following peripheral nerve surgery. Ultimately, we have not examined the diagnostic test accuracy of each modality head-to-head and this must be performed before comments about relatively accuracy and cost effectiveness can be made. Overall, we suggest that DTI might provide additional valuable information for the diagnosis, grading and management of (at least unclear or complex) patients with CTS. However, incorporating DTI in the real-world management of CTS would be difficult and require significant training for clinicians, changes to infrastructure and clinical pathways. None-the-less, we show that DTI yields unique information about the 'health' of the median nerve which could of significant clinical value. Initially, this technology could be used in patients with an unclear diagnosis or bilateral symptomatology, and those who don't improve after treatment.

We observed high statistical heterogeneity which has many potential explanations. The majority of the (statistical) heterogeneity was explained by the presence of CTS and it is plausible that the remainder is explained by the 'severity' of disease, which we were unable to capture. For example, we speculate that patients with more severe CTS (e.g., symptoms for years, resulting in profound demyelination, axonal loss and fibrosis) are likely to have lower FA and higher MD than patients with recent-onset mild CTS. Age also explained some of the observed heterogeneity and this is unsurprising, given that FA is known to fall in aging peripheral nerves ${ }^{64}$, just as it does in the white matter tracts of the brain ${ }^{65,66}$. This is because aging axons lose integrity, undergo demyelination and there is a simultaneous increase in extra-cellular fluid. Importantly, we showed that DTI metrics were sensitive to CTS after adjusting for age. Finally, in highly controlled and extreme conditions, user-specified factors ${ }^{67}$ such as the $\mathrm{SNR}^{68}$, b-value ${ }^{69,70}, \mathrm{~N}_{\mathrm{D}}{ }^{71,72}$, distortion correction pipelines ${ }^{73,74}$, tensor fitting methods ${ }^{68}$ and partial volume effects ${ }^{75}$ have been shown affect the DTI parameter estimates, which may explain some of the remaining heterogeneity. Although we could not explore the effects of all these factors, in general we observed that experimental conditions had little or no significant effect on the measured FA or MD. Therefore, despite the statistical heterogeneity, DTI appears to be reliably sensitive to the microstructural changes of the median nerve which occur in CTS.

There were no significant associations between FA and MD, and several core elements of the pulse sequence. Therefore, we suggest that clinicians and researchers wishing to acquire DTI could optimise their sequence as follows. As tensors are robust to varying b-values (in the hindered range) we suggest a b-value of $300-800 \mathrm{~mm}^{2} / \mathrm{s}$; smaller b-values enable a shorter TE, which improves SNR and mitigates the effects of T2 shine-through at the expense of less diffusion-weighting. Reducing the TE might also enable users to take advantage of other vendor-specific options to improve data quality and reduce distortions. Given that the median nerve has no crossing fibres to model, it is not tortuous (within or between voxels) and increasing the $\mathrm{N}_{\mathrm{D}}$ has little effect on simple tensor fitting ${ }^{76}$, we see no reason for the $\mathrm{N}_{\mathrm{D}}$ to exceed approximately 15 . The normal median nerve has a cross-sectional area of $9 \mathrm{~mm}^{2}(3.4 \mathrm{~mm} \text { diameter })^{77}$ and this increases with CTS ${ }^{78}$, so we recommend an in-plane resolution of approximately $1 \mathrm{~mm}^{2}$ to ensure that at least 1 voxel is not affected by partial voluming ${ }^{75}$. As that the median nerve is orthogonal to the imaging plane (if data are acquired axially), the slice thickness could be comfortably increased until there is adequate SNR because it appears to have little effect on the resultant metrics. Until work is published to show the exact relaxation properties (T1, T2, T2*) of the median nerve we suggest that TR is set to approximately $4500 \mathrm{~ms}$ to reduce scan time. Ideally, users specify an even number of signal averages (full datasets), divided equally between opposing phase-encoding directions (i.e. 1 signal acquisition blip-up and 1 blip-down or 2 averages blip-up and 2 blip-down, etc.) as this would allow offline concatenation and exploitation of the various corrections available in the FMRIB Software Library (FSL $)^{79}$. We advocate capturing such data via ssEPI because it is more time efficient than current implementations of rsEPI and distortions associated with ssEPI can be ameliorated using various softwares. It should be noted that thicker slices, longer repetition times and more signal averages are associated with lower estimates of $\mathrm{MD}$, if this is of importance to users.

Limitations. The main limitation of this study is the inherent and pervasive problem of CTS diagnosis which may have biased the findings. At present there is no internationally agreed diagnostic criteria for CTS and as such, there is clinical variation which is present in the includes studies. We planned to capture disease severity from the original studies, but this information was not available. As a matter of urgency, the community should work towards a consensus on objective criteria which constitute a diagnosis of CTS. Thereaftere, a reference standard for the diagnosis can be defined and this would enable studies of diagnostic test accuracy to be done, comparing DTI to other available tests such as electrophysiology, ultrasound and more.

It is widely known that diffusion metrics in the brain are strongly dependent on preprocessing pipelines (i.e., software $)^{79}$ but still there is no consensus on the minimum or indeed ideal suite of corrections to perform. This issue is compounded in the limb owing to an absence of research on the topic and hardware limitations. The majority of the included studies did not describe any form of distortion correction, how the diffusion data were reconstructed or how metrics were extracted from the median nerve. Before DTI can be used clinically, variations in these pipelines should be tested and a universal pipeline and standards for reporting diffusion data should be agreed by consensus.

Some readers will criticise our choice to pool estimates of FA and MD in the presence of high statistical heterogeneity. This was done because forest plots provide an important graphical representation of the variability of measurements in relation to experimental conditions (e.g., b-values and $\mathrm{N}_{\mathrm{D}}$ ), they summarise a large amount of information in an easy-to-interpret format and moreover, meta-regression facilitates the exploration of heterogeneity. 


\section{Conclusions}

We provide summary estimates of the normal FA and MD of the median nerve in asymptomatic adults. Furthermore, we show that diffusion throughout the length of the median nerve becomes more isotropic in patients with CTS, with the largest differences at the midpoint of the carpal tunnel at the level of the pisiform bone.

\section{Data availability}

The raw data are available via the Open Science Framework (https://osf.io/vqwkp/). The statistical syntax is available from the senior author (RGW) upon request.

Received: 8 July 2021; Accepted: 5 October 2021

Published online: 22 October 2021

\section{References}

1. Milone, M. T. et al. Analysis of expected costs of carpal tunnel syndrome treatment strategies. Hand 14(3), 317-323 (2019)

2. Neary, D. \& Eames, R. A. The pathology of ulnar nerve compression in man. Neuropathol. Appl. Neurobiol. 1(1), 69-88 (1975).

3. Pham, K. \& Gupta, R. Understanding the mechanisms of entrapment neuropathies. Neurosurg. Focus. 26(2), E7 (2009).

4. Nath, V. et al. Tractography reproducibility challenge with empirical data (TraCED): The 2017 ISMRM diffusion study group challenge. J. Magn. Reson. Imaging. 51(1), 234-249 (2020).

5. Vavasour, I. M. et al. Multicenter measurements of T 1 relaxation and diffusion tensor imaging: Intra and intersite reproducibility. J. Neuroimaging. 29(1), 42-51 (2019).

6. Prohl, A. K. et al. Reproducibility of structural and diffusion tensor imaging in the TACERN multi-center study. Front. Integr. Neurosci. 13, 1-15 (2019).

7. Kimura, M. et al. The reproducibility of measurements using a standardization phantom for the evaluation of fractional anisotropy (FA) derived from diffusion tensor imaging (DTI). Magn. Reson. Mater. Phys. Biol. Med. 2019, 15-19 (2019).

8. Cai, L. Y. et al. MASiVar: Multisite, multiscanner, and multisubject acquisitions for studying variability in diffusion weighted MRI. Magn. Reson. Med. 2021, 28926 (2021).

9. Heckel, A. et al. Peripheral nerve diffusion tensor imaging: Assessment of axon and myelin sheath integrity. PLoS ONE 10(6), e0130833 (2015).

10. Andersson, G. et al. In vivo diffusion tensor imaging, diffusion kurtosis imaging, and tractography of a sciatic nerve injury model in rat at 9.4T. Sci. Rep. 8(1), 12911 (2018).

11. Friedrich, P. et al. The relationship between axon density, myelination, and fractional anisotropy in the human corpus callosum. Cereb. Cortex. 30(4), 2042-2056 (2020).

12. Higgins, J. P. T. \& Green, S. (editors). Cochrane Handbook for Systematic Reviews of Interventions Version 5.1.0 [updated March 2011]. Cochrane Collab. (2011).

13. Page, M. J. et al. The PRISMA 2020 statement: An updated guideline for reporting systematic reviews. Syst. Rev. 10, 1 (2021).

14. McGuinness, L. \& Schmidt, L. medrxivr: Accessing and searching medRxiv and bioRxiv preprint data in R. J. Open Source Softw. 5(54), 2651 (2020).

15. Haddaway, N. R. Citationchaser: An R Package for Forward and Backward Citations Chasing in Academic Searching. Zenodo; 2021. https://zenodo.org/record/4533747. Accessed 15 Feb 2021.

16. Wade, R. G. et al. MRI for detecting root avulsions in traumatic adult brachial plexus injuries: A systematic review and metaanalysis of diagnostic accuracy. Radiology 293(1), 125-133 (2019).

17. Stein, D. et al. Diffusion tensor imaging of the median nerve in healthy and carpal tunnel syndrome subjects. J. Magn. Reson. Imaging. 29(3), 657-662 (2009).

18. Razek, A. A. K. A. et al. Diffusion tensor imaging of mild-moderate carpal tunnel syndrome: Correlation with nerve conduction study and clinical tests. Clin. Rheumatol. 36(10), 2319-2324 (2017).

19. Schmid, A. B. et al. Feasibility of diffusion tensor and morphologic imaging of peripheral nerves at ultra-high field strength. Radiology 53(12), 705-713 (2018).

20. Paniandi, V. et al. MR neurography of median nerve using diffusion tensor. Neurol. Asia. 2018, 9 (2018).

21. Wan, X. et al. Estimating the sample mean and standard deviation from the sample size, median, range and/or interquartile range. BMC Med. Res. Methodol. 14(1), 135 (2014).

22. Sterne, J. A. et al. ROBINS-I: A tool for assessing risk of bias in non-randomised studies of interventions. BMJ 355, 4919 (2016).

23. McGuinness, L. A. \& Higgins, J. P. T. Risk-of-bias VISualization (robvis): An R package and Shiny web app for visualizing risk-ofbias assessments. Res. Synth. Methods. 2020, 1411 (2020).

24. Higgins, J. P. T. \& Thompson, S. G. Quantifying heterogeneity in a meta-analysis. Stat. Med. 21(11), 1539-1558 (2002).

25. Viechtbauer, W. Conducting meta-analyses in R with the metafor package. J. Stat. Softw. 36, 3 (2010).

26. Haddaway, N. R. et al. EviAtlas: A tool for visualising evidence synthesis databases. Environ. Evid. 8(1), 22 (2019).

27. Barcelo, C. et al. 3-T MRI with diffusion tensor imaging and tractography of the median nerve. Eur. Radiol. 1, 7 (2019).

28. Brienza, M. et al. 3T diffusion tensor imaging and electroneurography of peripheral nerve: A morphofunctional analysis in carpal tunnel syndrome. J. Neuroradiol. 41(2), 124-130 (2014).

29. Koh, S. H. et al. A comparison of the performance of anatomical MRI and DTI in diagnosing carpal tunnel syndrome. Eur. J. Radiol. 83(11), 2065-2073 (2014).

30. Edward, R. et al. A study of diffusion tensor imaging of median nerve in diabetic peripheral neuropathy. Egypt. J. Neurol. Psychiatry Neurosurg. 56(1), 172 (2020).

31. Filli, L. et al. Accelerated magnetic resonance diffusion tensor imaging of the median nerve using simultaneous multi-slice echo planar imaging with blipped CAIPIRINHA. Eur. Radiol. 26(6), 1921-1928 (2016).

32. Guggenberger, R. et al. Assessment of median nerve with MR neurography by using diffusion-tensor imaging: Normative and pathologic diffusion values. Radiology 265(1), 194-203 (2012).

33. Wang, I.-M., Huang, H.-W., Chen, P.-Y., Tsai, H.-M. \& Liu, Y. S. Carpal tunnel syndrome assessed with diffusion tensor imaging: Comparison with electrophysiological studies of patients and healthy volunteers. Radiology 81(11), 3378-3383 (2012).

34. Klauser, A. S. et al. Carpal tunnel syndrome assessment with diffusion tensor imaging: Value of fractional anisotropy and apparent diffusion coefficient. Eur. Radiol. 28, 1111-1117 (2017).

35. Breckwoldt, M. O. et al. Diffusion tensor imaging adds diagnostic accuracy in magnetic resonance neurography. Invest. Radiol. 50(8), 7 (2015)

36. Andreisek, L. M., Kassner, A., Tomlinson, G. \& Sussman, M. S. Diffusion tensor imaging and fiber tractography of the median nerve at 1.5T: Optimization of b value. Eur. Radiol. 38(1), 51-59 (2008).

37. Hiltunen, J. et al. Diffusion tensor imaging and tractography of distal peripheral nerves at 3 T. Clin. Neurophysiol. 116(10), 2315-2323 (2005). 
38. Kabakci, N. et al. Diffusion tensor imaging and tractography of median nerve: Normative diffusion values. Am. J. Roentgenol. 189(4), 923-927 (2007).

39. Khalil, C. et al. Diffusion tensor imaging and tractography of the median nerve in carpal tunnel syndrome: Preliminary results. Eur. Radiol. 18(10), 2283-2291 (2008).

40. Tasdelen, N. et al. Diffusion tensor imaging in carpal tunnel syndrome. Diagn. Interv. Radiol. 2, 60-66 (2011).

41. Zhou, Y. et al. Diffusion tensor imaging of forearm nerves in humans. J. Magn. Reson. Imaging. 36(4), 920-927 (2012).

42. Guggenberger, R. et al. Diffusion tensor imaging of the median nerve at 3.0T using different MR scanners: Agreement of FA and ADC measurements. Eur. J. Radiol. 82(10), e590-e596 (2013).

43. Naraghi, A. et al. Diffusion tensor imaging of the median nerve before and after carpal tunnel release in patients with carpal tunnel syndrome: feasibility study. Skelet. Radiol. 42(10), 1403-1412 (2013).

44. Antoine, L., Le Viet, D., Maier, M. A. \& Drapé, J.-L. Diffusion tensor imaging of the median nerve in recurrent carpal tunnel syndrome: Initial experience. Skelet. Radiol. 23(11), 3115-3123 (2013).

45. Giray, C. S., Alis, D., Samanci, C., Kandemirli, G. C. \& Adatepe, N. U. M. K. Evaluation of median nerve by shear wave elastography and diffusion tensor imaging in carpal tunnel syndrome. Eur. J. Radiol. 101, 59-64 (2018).

46. Yao, L. \& Gai, N. Median nerve cross-sectional area and MRI diffusion characteristics: Normative values at the carpal tunnel. Skeletal Radiol. 38(4), 355-361 (2009).

47. Kwon, B. C., Koh, S. H. \& Hwang, S. Y. Optimal parameters and location for diffusion-tensor imaging in the diagnosis of carpal tunnel syndrome: A prospective matched case-control study. Am. J. Roentgenol. 204(6), 1248-1254 (2015).

48. Erika, H., Numminen, J., Lindfors, N., Göransson, H. \& Hari, R. J. K. Pre- and post-operative diffusion tensor imaging of the median nerve in carpal tunnel syndrome. J. Radiol. Nucl. Med. 22(6), 1310-1319 (2012).

49. Wafaie, A. M. et al. Role of diffusion tensor imaging in carpal tunnel syndrome: A case control comparative study to electrophysiological tests and clinical assessment. Egypt. J. Radiol. Nucl. Med. 49(4), 1068-1075 (2018).

50. Bulut, H. T. et al. The diagnostic and grading value of diffusion tensor imaging in patients with carpal tunnel syndrome. Acad. Radiol. 21(6), 767-773 (2014).

51. Ding, W. Q. et al. Three-dimensional display of peripheral nerves in the wrist region based on MR diffusion tensor imaging and maximum intensity projection post-processing. Eur. J. Radiol. 84(6), 1116-1127 (2015).

52. Taner, Y. H., Ekmekçi, B., Sürücü, G. D. \& Karabiber, M. A. B. Use of diffusion tensor imaging for nonsurgical treatments of carpal tunnel syndrome. Eur. J. Radiol. 50(6), 950-955 (2014).

53. Pridmore, M. D. et al. Initial findings in traumatic peripheral nerve injury and repair with diffusion tensor imaging. Ann. Clin. Transl. Neurol. 8(2), 332-347 (2021).

54. Simon, J. et al. The accuracy of the scratch collapse test performed by blinded examiners on patients with suspected carpal tunnel syndrome assessed by electrodiagnostic studies. J. Hand Surg. 42, 1-5 (2017).

55. D'Arcy, C. A. Does this patient have carpal tunnel syndrome?. JAMA 283(23), 3110 (2000).

56. Lu, Y.-T., Deol, A. K. \& Sears, E. D. The association between electrodiagnostic severity and treatment recommendations for carpal tunnel syndrome. J. Hand Surg. 46(2), 92-98 (2021).

57. Roll, S. C., Case-Smith, J. \& Evans, K. D. Diagnostic accuracy of ultrasonography vs electromyography in carpal tunnel syndrome: A systematic review of literature. Ultrasound Med. Biol. 37(10), 1539-1553 (2011).

58. Mackenzie, S. P. et al. Carpal tunnel decompression in patients with normal nerve conduction studies. J. Hand Surg. Eur. 45(3), 260-264 (2020).

59. Erickson, M., Lawrence, M. \& Lucado, A. The role of diagnostic ultrasound in the examination of carpal tunnel syndrome: An update and systematic review. J. Hand Ther. 1, S0894 (2021).

60. Fisse, A. L. et al. Cross-sectional area reference values for peripheral nerve ultrasound in adults: A systematic review and metaanalysis-Part I: Upper extremity nerves. Eur. J. Neurol. 28(5), 1684-1691 (2021).

61. Torres-Costoso, A. et al. Accuracy of ultrasonography for the diagnosis of carpal tunnel syndrome: A systematic review and metaanalysis. Arch. Phys. Med. Rehabil. 99(4), 758-765.e10 (2018).

62. Chen, S. et al. Electrodiagnostic reference values for upper and lower limb nerve conduction studies in adult populations: AANEM Practice Topic. Muscle Nerve. 54(3), 371-377 (2016).

63. Anon. NIHR 2020 Investigation and Intervention Tariff 1.2.pdf.

64. Kronlage, M. et al. Peripheral nerve diffusion tensor imaging (DTI): Normal values and demographic determinants in a cohort of 60 healthy individuals. Eur. Radiol. 28(5), 1801-1808 (2018).

65. Yap, Q. J. et al. Tracking cerebral white matter changes across the lifespan: Insights from diffusion tensor imaging studies. J. Neural Transm. 120(9), 1369-1395 (2013).

66. Watanabe, M. et al. Age-related apparent diffusion coefficient changes in the normal brain. Radiology 266(2), 575-582 (2013).

67. Helmer, K. G. et al. Multi-site study of diffusion metric variability: Effects of site, vendor, field strength, and echo time on regionsof-interest and histogram-bin analyses. Physiol. Behav. 176, 97882U (2016).

68. Jones, D. K. \& Basser, P. J. "Squashing peanuts and smashing pumpkins": How noise distorts diffusion-weighted MR data. Magn. Reson. Med. 52(5), 979-993 (2004).

69. Schilling, K. G., et al. Effects of b-value and number of gradient directions on diffusion MRI measures obtained with Q-ball imaging. in Proc. SPIE Int. Soc. Opt. Eng. (Styner, M. A. \& Angelini, E. D., eds) (2017).

70. Qin, W. et al. Effects of echo time on diffusion quantification of brain white matter at 1.5T and 3.0T. Magn. Reson. Med. 61(4), 755-760 (2009).

71. Haakma, W. et al. Diffusion tensor imaging of peripheral nerves in non-fixed post-mortem subjects. Forensic Sci. Int. 263, 139-146 (2016).

72. Giannelli, M. et al. Dependence of brain DTI maps of fractional anisotropy and mean diffusivity on the number of diffusion weighting directions. J. Appl. Clin. Med. Phys. 11(1), 176-190 (2010).

73. Taylor, P. A. et al. Assessing the performance of different DTI motion correction strategies in the presence of EPI distortion correction. Hum. Brain Mapp. 37(12), 4405-4424 (2016).

74. Haddad, S. M. H. et al. Comparison of quality control methods for automated diffusion tensor imaging analysis pipelines. PLoS ONE 14(12), e0226715 (2019).

75. Vos, S. B. et al. Partial volume effect as a hidden covariate in DTI analyses. Neuroimage 55(4), 1566-1576 (2011).

76. Ni, H. et al. Effects of number of diffusion gradient directions on derived diffusion tensor imaging indices in human brain. Am. J. Neuroradiol. 27(8), 1776-1781 (2006).

77. Klauser Ethan, J. et al. Carpal tunnel syndrome assessment with US: Value of additional cross-sectional area measurements of the median nerve in patients versus healthy volunteers. Am. J. Neuroradiol. 250(1), 171-177 (2008).

78. Roomizadeh, P. et al. Ultrasonographic assessment of carpal tunnel syndrome severity: A systematic review and meta-analysis. Am. J. Phys. Med. Rehabil. 98(5), 373-381 (2019).

79. Brun, L. et al. Diffusion MRI: Assessment of the impact of acquisition and preprocessing methods using the BrainVISA-diffuse toolbox. Front. Neurosci. 13, 536 (2019). 


\section{Author contributions}

D.R. co-authored and registered the protocol, performed the searches, screened articles, extracted data, performed the risk of bias assessments and co-authored the manuscript. F.R. screened articles, extracted data, performed the risk of bias assessments and co-authored the manuscript. J.R. screened articles, extracted data, performed the risk of bias assessments and co-authored the manuscript. R.G.W. conceived the study, co-authored the protocol, supervised the searches and citation chasing, checked all the extracted data for accuracy, performed the statistical analyses, created the artwork and co-authored the manuscript. All authors had full access to all the data in the study and had final responsibility for the decision to submit for publication.

\section{Funding}

Ryckie Wade is a Doctoral Research Fellow funded by the National Institute for Health Research (NIHR, DRF2018-11-ST2-028). The views expressed are those of the author(s) and not necessarily those of the United Kingdom's National Health Service, NIHR or Department of Health.

\section{Competing interests}

The authors declare no competing interests.

\section{Additional information}

Supplementary Information The online version contains supplementary material available at https://doi.org/ 10.1038/s41598-021-00353-z.

Correspondence and requests for materials should be addressed to R.G.W.

Reprints and permissions information is available at www.nature.com/reprints.

Publisher's note Springer Nature remains neutral with regard to jurisdictional claims in published maps and institutional affiliations.

(c) (i) Open Access This article is licensed under a Creative Commons Attribution 4.0 International cc) License, which permits use, sharing, adaptation, distribution and reproduction in any medium or format, as long as you give appropriate credit to the original author(s) and the source, provide a link to the Creative Commons licence, and indicate if changes were made. The images or other third party material in this article are included in the article's Creative Commons licence, unless indicated otherwise in a credit line to the material. If material is not included in the article's Creative Commons licence and your intended use is not permitted by statutory regulation or exceeds the permitted use, you will need to obtain permission directly from the copyright holder. To view a copy of this licence, visit http://creativecommons.org/licenses/by/4.0/.

(C) The Author(s) 2021 